positive cases. TMAs included 535 cases reported within our institution (DHM Pathology) over a 10 year period. Four cases showed moderate to strong positivity with ALK immunohistochemistry. Cases included a pleomorphic rhabdomyosarcoma (FOXO1-/SS18-/EWSR1-/MDM2-; 1/11), metastatic Ewing's sarcoma (EWSR1 FISH-proven; 1/16), an undifferentiated pleomorphic sarcoma (1/68) and uterine myxoid leiomyosarcoma (1/118). No significant staining was identified in myxofibrosarcomas ( $n=97$ ), well-differentiated liposarcomas (LPS)/ atypical lipomatous tumours $(n=95)$, dedifferentiated LPS $(n=39)$, myxoid LPS $(n=28)$, angiosarcomas $(n=17)$, synovial sarcomas $(n=17)$ and pleomorphic LPS $(n=11)$. ALK gene fusion analysis is pending but this preliminary data confirms the high specificity and sensitivity of the ALK antibody with negative stain in $99.25 \%$ of cases, restricting the necessity of molecular testing to $0.75 \%$ of cases. If ALK translocations are confirmed, this broadens the range of mesenchymal tumours in which ALK rearrangements may be present.

\section{CLINICAL IMPLICATIONS OF DISCORDANT MASSARRAY AND SANGER SEQUENCING RESULTS IN CYSTIC FIBROSIS NEWBORN SCREENING}

$\underline{\text { Dylan A. Mordaunt }}^{1,2}$, Emily Button ${ }^{1}$, Tristan Hardy ${ }^{1}$, $\overline{\text { Andrew Dubowsky }}^{1}$, Mark Holloway ${ }^{1}$, Sarah Schenscher ${ }^{3}$, Drago Bratkovic ${ }^{3}$, Janice Fletcher ${ }^{1}$

${ }^{1}$ Genetics and Molecular Pathology, SA Pathology, SA, Australia; ${ }^{2}$ Department of Paediatrics, School of Medicine,
University of Adelaide, SA, Australia; and ${ }^{3}$ Department of Metabolic Medicine, Women's and Children's Health Network, SA, Australia

Background: Agena's MassArray platform is used for high throughput genotyping in our service. This method combines primer extension termination polymerase chain reaction (PCR) with mass spectrometry (MADI-TOF) and proprietary genotype calling algorithms.

Method: The Agena iPLEX CFTR 74 v3 off-the-shelf assay is used as a 'common variant screen' for cystic fibrosis (CFTR) genotyping, including infants with an immunoreactive trypsin in the top $1 \%$ on newborn blood spot screening. Variants detected by this assay are not routinely confirmed by Sanger sequencing.

Case report: Cascade testing identified a variant called by the MassArray platform as c.2175dupA to be discordant with capillary electrophoresis sequencing, which identified this as c. $2173 \mathrm{G}>\mathrm{A}$. Many of the variants on this screening panel are rare in our experience. The pathogenic c.2175dupA variant had not been previously detected in clinical testing in our service. The c. $2173 \mathrm{G}>\mathrm{A}$ variant is classified as a variant of unknown significance and would not usually be reported in a newborn screening patient.

Discussion: A primer extension product with a specific mass to charge ratio can detect different PCR products. The presence of rare SNPs is listed as a method limitation, but in this Case, resulted in variant re-classification. 